\title{
Quantitative Inhibitor Fingerprinting of Metalloproteases Using Small Molecule
}

\section{Microarrays}

Mahesh Uttamchandani ${ }^{\ddagger}$, Wei L. Lee ${ }^{\ddagger}$, Jun Wang ${ }^{\S}$, Shao Q. Yao ${ }^{\ddagger \S \uparrow *}$

Department of Biological Sciences, Department of Chemistry and NUS MedChem Program of the Office of Life Sciences, 3 Science Drive 3, National University of Singapore,

Singapore 117543

E-Mail: chmyaosq@nus.edu.sg

Running Title: Inhibitor Fingerprinting of Metalloproteases

Keywords: Metalloproteases, Hydroxamate inhibitors, Microarrays, High-throughput screening, Inhibitor fingerprinting, Inhibitors, Catalomics

\footnotetext{
${ }^{\ddagger}$ Department of Biological Sciences

$\S$ Department of Chemistry

" NUS MedChem Program of the Office of Life Sciences
} 


\section{Supplemental Materials and Methods:}

Preparation of slides. $25 \mathrm{~mm} \times 75 \mathrm{~mm}$ glass slides (Sigma-Aldrich) were cleaned in piranha solution (sulfuric acid/hydrogen peroxide, 7:3). An amine functionality was incorporated onto the slides by silanization using a solution of 3\% (aminopropyl)triethoxysilane in $2 \%$ water and $95 \%$ ethanol. After 1-2 $\mathrm{h}$ incubation, the slides were washed with ethanol and cured at $150^{\circ} \mathrm{C}$ for at least $2 \mathrm{~h}$. The resulting amine slides were incubated in a solution of $180 \mathrm{mM}$ succinic anhydride in DMF for 30 min before being transferred to a boiling water bath for $2 \mathrm{~min}$. The slides were rinsed with ethanol and dried under a stream of nitrogen. The carboxylic acid derivatized slide surface was activated with a solution of 100 $\mathrm{mM}$ of HBTU (O-Benzotriazole-N,N,N',N'-tetramethyl-uronium-hexafluoro-phosphate), $200 \mathrm{mM}$ DIEA, and $100 \mathrm{mM} N$-hydroxysuccinimide in DMF, thus generating the NHS-derivatized slides. These surfaces were reacted with a solution of $1 \mathrm{mg} / \mathrm{mL}$ avidin in $10 \mathrm{mM} \mathrm{NaHCO}$ (pH 9) for 40 minutes, washed with water, air dried. The unreacted NHS groups were quenched with a solution of $2 \mathrm{mM}$ aspartic acid in a $0.5 \mathrm{M} \mathrm{NaHCO}_{3}$ buffer, $\mathrm{pH}$ 9. These slides were washed with water, dried and stored at $4^{\circ} \mathrm{C}$, ready for printing.

Data extraction and analysis. Microarray data was extracted using the ArrayWoRx software. Values from duplicate points were background subtracted and averaged. Raw signals arising from the inactive channel were thereafter deducted from the active channel to give the normalized activity-dependent results for each experiment. Independent data from the reciprocal experiments was then combined by averaging. Points that were inconsistent across both channels were removed ( $>1$ standard deviation fold variation between the two experiments). The dataset showed a good consistency across duplicated experiments (Pearson correlation, $r>0.8$, across all 1,400 datapoints). The combined dataset was scaled from $0 \%-100 \%$ by fitting a maximum value within each enzyme dataset to $100 \%$ (mean +1 standard deviation point). The dataset was presented in both 3-D cube plots, using the Graphis software (Kylebank, UK), and as colored heatmaps, using the Treeview software (http://rana.lbl.gov/EisenSoftware.htm). Position specific scoring matrix analysis was performed using software developed by Stephen Shaw (sshaw@nih.gov) and obtained and used with permission. ${ }^{1}$ The dataset was also subjected to cluster analysis to correlate specificity information from the library into hierarchical contributions, providing insights of inhibitor-dependent similarities across the enzyme set. Averaged linkage clustering was performed using the Systat v11.0 software (San Jose, CA, USA). For comparison, the active site domains were retrieved from MEROPS ${ }^{2}$ and aligned using ClustalW. ${ }^{3}$ Venn diagrams were generated using the Venn Diagram Generator (http://www.pangloss.com/seidel/Protocols/venn.cgi). The complete dataset obtained is provided as a supplementary Microsoft Excel file.

Microarray preparation. Hydroxamate inhibitors stock solutions were prepared to 2.5 mM in 50\% DMSO with 50\% PBS, and were distributed in 384-well plates. Slides were spotted on an ESI SMA arrayer (Ontario, Canada) with the printhead installed with 8 Stealth SMP8B Microspotting pins (Telechem, U.S.A.). Spots generated were of approximately $350 \mu \mathrm{m}$ diameter and were printed with a spot-spot spacing of $450 \mu \mathrm{m}$. The pins were rinsed in between samples using two cycles of wash (for 10 s) and sonication (for $10 \mathrm{~s}$ ) in reservoirs containing $70 \%$ ethanol followed by drying under reduced pressure (for $10 \mathrm{~s}$ ). The slides were allowed to stand for $1 \mathrm{~h}$ on the printer platform and stored at $4^{\circ} \mathrm{C}$ until use. Before incubation with the labeled protein, the slides were rinsed with PBS (pH 7.4) for 20 min and blocked with PBS containing 1\% BSA for $1 \mathrm{~h}$. 
Microplate-based screen of sub-library with thermolysin. We performed screening on microplates to evaluate the relative potencies of thermolysin against a subset of the library. We used the 400member sub-library presenting leucine in the $\mathrm{P}_{1}$ ' position. Assays were performed under optimized conditions in 384-well black polypropylene plates and the reaction components were assembled robotically using an 8-channel robotic dispensing system (Precision XS, Biotek, Vermont, USA). Cleavage of bodipy-labeled casein substrate (prepared to a final reaction concentration of $4.4 \mu \mathrm{g} / \mathrm{ml}$ ) (Molecular Probes, Invitrogen, USA) was monitored over $1 \mathrm{~h}$ in the presence of thermolysin and each of the inhibitor (final concentration: $660 \mathrm{nM}$ ). Each reaction was performed in a $50 \mu$ l volume, buffered in PBS (pH 7.4). Upon enzyme addition to $1.8 \mu \mathrm{M}$ in the final step to initiate the reaction, the plates were incubated at $37^{\circ} \mathrm{C}$ for $30 \mathrm{~min}$, before being queried for endpoint fluorescence on a SpectraMax ${ }^{\mathrm{TM}}$ Gemini XS fluorescence plate reader $\left(\lambda_{\text {ex/em }}=490 / 528 \mathrm{~nm}\right)$ (Molecular Devices, USA) in staggered runs. Plate readings were exported from the scanner SoftmaxPro software into Microsoft Excel. Raw results were normalized by subtracting readings from the initial uncleaved substrate and inhibitor backgrounds. Data points from each plate were benchmarked against positive controls (these were essentially uninhibited samples, with enzymes assayed without inhibitor, replicated at 5 wells per plate). The relative potencies of each inhibitor were calculated from the normalized data through the following relationship:

$$
\text { Inhibition potency of } \mathrm{x}=\left(1-\frac{\text { Measured intensity, } \mathrm{x}}{\text { Uninhibited intensity }}\right) \times 100 \%
$$

The data was presented as colored heatmaps using the treeview (http://rana.lbl.gov/EisenSoftware.hstm) software.

$K_{\mathbf{D}}$ analysis using SPR. Surface plasmon resonance measurements were performed on a Biacore 3000 instrument (Biacore AB, Uppsala, Sweden). CM-5 chips were functionalized with avidin using standard procedures. The relevant test peptides were immobilized to approximately 400 response units (RUs). An adjacent chip flow cell was left unimmobilized for use as a reference for subtraction. Eight different concentrations of enzyme were applied in duplicate, in a 3-fold dilution series ranging from 5 $\mu \mathrm{M}$ to $2.3 \mathrm{nM}$. A buffer comprising $10 \mathrm{mM}$ HEPES (pH 7.4), $150 \mathrm{mM} \mathrm{NaCl}$ and $0.01 \%$ Tween 20 was used under a flow rate of $30 \mu \mathrm{l} / \mathrm{min}$. The chip surface was regenerated between samples with injections of $0.1 \mathrm{M}$ glycine ( $\mathrm{pH}$ 2). The resulting data was analyzed using the BiaEvaluation software (Biacore AB, Uppsala, Sweden) under the 1:1 (Langmuir) binding model. Proper maintenance was performed to ensure that the instrument was kept in good running condition. The integrated flow cell and fluidic system was regularly washed, sanitized and desorbed using standard cleansing reagents, as recommended. 


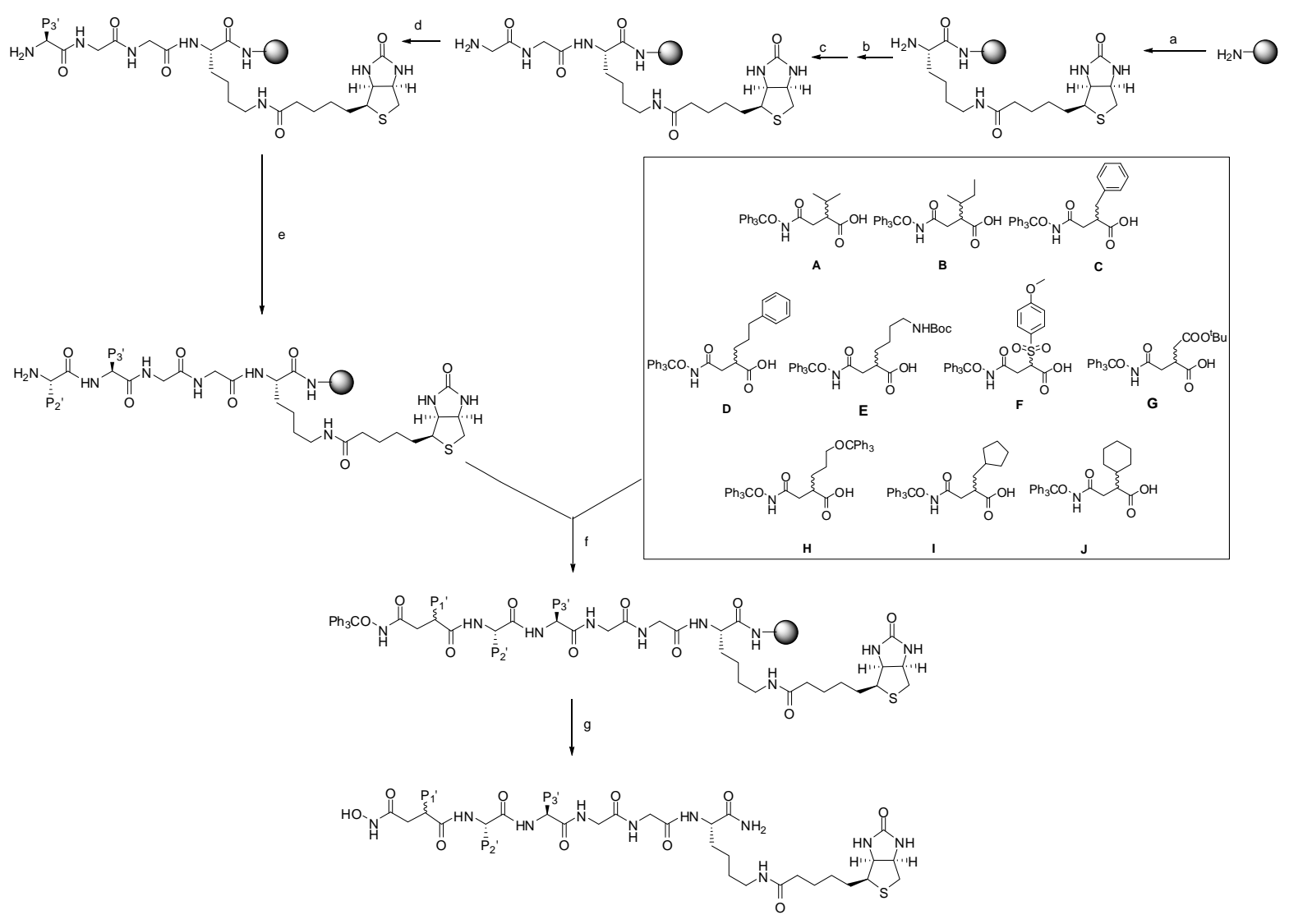

Scheme S1. Reagents and conditions for synthesis of 1,400-member hydroxamate library (a)i:FmocLys(Biotin)-OH, HOBt, HBTU, DIEA, DMF, 12 hrs; ii: 20\% piperidine/DMF, 2hrs; (b) i:Fmoc-GlyOH, HOBt, HBTU, DIEA, 6 hrs; ii: 20\% piperidine/DMF, 2hrs; (c) i:Fmoc-Gly-OH, HOBt, HBTU, DIEA, 6 hrs; ii: 20\% piperidine/DMF, 2hrs; (c) i:Fmoc-AA3-OH, HOBt, DIC, DIEA, 8 hrs; ii: 20\% piperidine/DMF, 2hrs; (d) i:Fmoc-AA2-OH, HOBt, HBTU, DIEA, 8 hrs; ii: 20\% piperidine/DMF, 2hrs; (e) i: CPh3ONH-Suc(2-P1')-COOH, 1\% Fmoc-ACC-COOH, HATU, 2,4,6-collidine, 12 hrs; ii: 20\% piperidine/DMF, 2hrs; (f) 95\% TFA/ 5\% TIS, 2hrs. 
Table S1. The classification of the panel of 4 metalloproteases used in this study. The enzymes in our panel were divided across two broad clans MA(E) and MC clans of metalloproteases, which differentiates the endopeptidases from the exopeptidase, as well as categories the enzymes according to the conserved active site motifs.

\begin{tabular}{lllc}
\multicolumn{1}{c}{ Enzyme } & \multicolumn{1}{c}{ Source } & \multicolumn{1}{c}{ Classification } & $\begin{array}{c}\text { Conserved } \\
\text { catalytic motif }\end{array}$ \\
\hline Thermolysin & $\begin{array}{l}\text { Bacillus } \\
\text { thermoproteolyticus }\end{array}$ & Clan MA(E) >> Family M4 & HEXXH \\
Anthrax LF & $\begin{array}{l}\text { Bacillus anthracis } \\
\text { Clostridium } \\
\text { histolyticum }\end{array}$ & Clan MA(E) >> Family M34 & HEXXH \\
Collagenase colA & Clan MA(E) >> Family M9 & HEXXH \\
Carboxypeptidase A1 & Clan MC >> Family M14 & HXXE
\end{tabular}

Table S2. Library design for 1400-member hydroxamate peptides. The 20 natural amino acids were used in the P2' and P3' position for in the Leu library, whilst a representative set of 10 amino acids (Nonpolar: Ala, Leu, Phe, Trp, Charged polar: Glu, Lys, His and Uncharged polar: Gln, Ser, Tyr) was utilized for permuting all other sublibraries.

\begin{tabular}{|c|c|c|c|}
\hline $\mathrm{P}_{1}^{\prime}$ & $\mathrm{P}_{2}^{\prime}$ & $\mathrm{P}_{3}{ }^{\prime}$ & Sub-Library Size \\
\hline $\mathbf{L}$ & $\begin{array}{l}20 \text { natural amino } \\
\text { acids }\end{array}$ & $\begin{array}{l}20 \text { natural amino } \\
\text { acids }\end{array}$ & 400 members \\
\hline $\begin{array}{c}\mathbf{I}, \mathbf{V}, \mathbf{F},{ }_{1} \mathbf{F}, \mathbf{K} \\
\mathbf{S}_{\mathbf{f}}, \mathbf{D},{ }_{1} \mathbf{S}, \mathbf{C}_{\mathbf{p}}, \mathbf{C}_{\mathbf{h}}\end{array}$ & $\begin{array}{l}10 \text { natural amino } \\
\text { acids }\end{array}$ & $\begin{array}{l}10 \text { natural amino } \\
\text { acids }\end{array}$ & 1000 members \\
\hline
\end{tabular}


Table S3. Selective inhibitors uncovered from the top-100 inhibitor lists. Inhibitors listed (in order of name) reflect the specific inhibitors that were exclusive to respective metalloproteases from within the top 100 inhibitor list. Identities are defined as $\mathrm{P}_{1}{ }^{\prime}-\mathrm{P}_{2}{ }^{\prime}-\mathrm{P}_{3}{ }^{\prime}$. Inhibitors in bold were further characterized with quantitative measurements.

\begin{tabular}{|c|c|c|c|c|c|}
\hline No. & Thermolysin & Collagenase & Carboxypeptidase & Ant & \\
\hline 1 & L-A-R & D-F-W & $\mathrm{C}_{\mathrm{p}}-\mathrm{F}-\mathrm{A}$ & $\mathrm{C}_{\mathrm{h}}-\mathrm{F}-\mathrm{S}$ & ${ }_{1} \mathrm{~F}-\mathrm{E}-\mathrm{Y}$ \\
\hline 2 & F-L-W & $\mathrm{C}_{\mathrm{p}}-\mathrm{H}-\mathrm{F}$ & $\mathrm{C}_{\mathrm{p}}-\mathrm{F}-\mathrm{L}$ & $\mathrm{C}_{\mathrm{h}}-\mathrm{K}-\mathrm{L}$ & ${ }_{1}$ F-F-L \\
\hline 3 & L-A-C & $\mathrm{C}_{\mathrm{p}}-\mathrm{Q}-\mathrm{Q}$ & $\mathrm{C}_{\mathrm{p}}-\mathrm{F}-\mathrm{Y}$ & $\mathrm{C}_{\mathrm{h}}-\mathrm{K}-\mathrm{W}$ & ${ }_{1} \mathrm{~F}-\mathrm{L}-\mathrm{K}$ \\
\hline 4 & L-A-K & F-L-A & $\mathrm{C}_{\mathrm{p}}-\mathrm{W}-\mathrm{A}$ & $\mathrm{C}_{\mathrm{h}}-\mathrm{L}-\mathrm{W}$ & ${ }_{1} \mathrm{~F}-\mathrm{L}-\mathrm{S}$ \\
\hline 5 & L-A-P & F-Y-A & $\mathrm{C}_{\mathrm{p}}-\mathrm{W}-\mathrm{Q}$ & $\mathrm{C}_{\mathrm{h}}-\mathrm{Q}-\mathrm{L}$ & L-F-M \\
\hline 6 & L-A-Q & F-Y-S & $\mathrm{C}_{\mathrm{p}}-\mathrm{W}-\mathrm{S}$ & $\mathrm{C}_{\mathrm{h}}-\mathrm{S}-\mathrm{S}$ & ${ }_{1} \mathrm{~F}-\mathrm{Q}-\mathrm{A}$ \\
\hline 7 & L-A-S & I-E-E & $\mathrm{C}_{\mathrm{p}}-\mathrm{Y}-\mathrm{L}$ & $\mathrm{C}_{\mathrm{h}}-\mathrm{W}-\mathrm{K}$ & F-Q-K \\
\hline 8 & L-A-V & I-L-L & F-F-F & $C_{h}-Y-A$ & ${ }_{1} \mathrm{~F}-\mathrm{Q}-\mathrm{S}$ \\
\hline 9 & L-H-C & $\mathrm{I}-\mathrm{Q}-\mathrm{L}$ & F-F-Q & $\mathrm{C}_{\mathrm{h}}-\mathrm{Y}-\mathrm{K}$ & ${ }_{1} \mathrm{~F}-\mathrm{S}-\mathrm{Y}$ \\
\hline 10 & L-H-R & I-Q-W & F-F-Y & $\mathrm{C}_{\mathrm{h}}-\mathrm{Y}-\mathrm{S}$ & L-H-L \\
\hline 11 & L-I-K & I-W-S & F-H-W & $\mathrm{C}_{\mathrm{p}}-\mathrm{K}-\mathrm{Y}$ & L-I-Y \\
\hline 12 & L-I-P & $\mathrm{I}-\mathrm{W}-\mathrm{Y}$ & F-Y-L & $\mathrm{C}_{\mathrm{p}}-\mathrm{L}-\mathrm{Q}$ & L-P-F \\
\hline 13 & L-L-F & $\mathrm{K}-\mathrm{K}-\mathrm{F}$ & F-Y-Q & $C_{p}-L-S$ & S-A-S \\
\hline 14 & L-L-P & K-W-S & I-F-A & $C_{p}-Y-S$ & ${ }_{1} \mathrm{~S}-\mathrm{L}-\mathrm{S}$ \\
\hline 15 & L-M-K & K-Y-L & $\mathrm{I}-\mathrm{F}-\mathrm{Q}$ & $C_{p}-Y-Y$ & ${ }_{1} S-S-E$ \\
\hline 16 & L-M-Y & L-C-L & I-K-L & D-K-A & L-S-W \\
\hline 17 & L-P-C & L-C-T & I-L-W & D-K-Q & ${ }_{1} S-Y-Q$ \\
\hline 18 & L-Q-K & ${ }_{1} F-E-W$ & $\mathrm{~K}-\mathrm{W}-\mathrm{F}$ & D-Q-Q & S-Y-S \\
\hline 19 & L-Q-P & L-F-F & K-W-W & $D-W-E$ & $\mathrm{~L}-\mathrm{V}-\mathrm{W}$ \\
\hline 20 & L-Q-W & L-F-G & ${ }_{1} \mathrm{~F}-\mathrm{A}-\mathrm{W}$ & D-W-L & L-W-K \\
\hline 21 & L-R-A & ${ }_{1} \mathrm{~F}-\mathrm{L}-\mathrm{Y}$ & ${ }_{1} \mathrm{~F}-\mathrm{F}-\mathrm{A}$ & D-W-Q & L-Y-A \\
\hline 22 & L-R-Q & ${ }_{1} \mathrm{~F}-\mathrm{S}-\mathrm{L}$ & ${ }_{1} \mathrm{~F}-\mathrm{F}-\mathrm{Y}$ & F-E-L & L-Y-V \\
\hline 23 & $\mathrm{~L}-\mathrm{V}-\mathrm{A}$ & L-H-V & ${ }_{1} \mathrm{~F}-\mathrm{H}-\mathrm{L}$ & F-H-Q & $\mathrm{S}_{\mathrm{f}}-\mathrm{F}-\mathrm{L}$ \\
\hline 24 & L-V-K & L-H-W & ${ }_{1} \mathrm{~F}-\mathrm{H}-\mathrm{W}$ & F-L-K & $S_{f}-K-E$ \\
\hline 25 & L-V-R & L-M-G & L-F-L & F-Q-F & $\mathrm{S}_{\mathrm{f}}-\mathrm{L}-\mathrm{Q}$ \\
\hline 26 & L-W-F & IS-A-A & ${ }_{1} \mathrm{~F}-\mathrm{Q}-\mathrm{H}$ & $\mathrm{I}-\mathrm{A}-\mathrm{Y}$ & $\mathrm{S}_{\mathrm{f}}-\mathrm{Q}-\mathrm{S}$ \\
\hline 27 & L-W-I & IS-F-W & ${ }_{1} \mathrm{~F}-\mathrm{Q}-\mathrm{W}$ & I-F-L & $\mathrm{S}_{\mathrm{f}}-\mathrm{W}-\mathrm{Q}$ \\
\hline 28 & L-W-M & L-Y-G & ${ }_{1} \mathrm{~F}-\mathrm{W}-\mathrm{E}$ & I-L-F & V-F-F \\
\hline 29 & & $\mathrm{~S}_{\mathrm{f}}-\mathrm{S}-\mathrm{K}$ & ${ }_{1} \mathrm{~F}-\mathrm{W}-\mathrm{H}$ & I-Q-Q & V-F-K \\
\hline 30 & & V-S-L & ${ }_{1} \mathrm{~F}-\mathrm{W}-\mathrm{Q}$ & $\mathrm{I}-\mathrm{S}-\mathrm{Q}$ & V-H-F \\
\hline 31 & & V-Y-L & ${ }_{1} \mathrm{~F}-\mathrm{Y}-\mathrm{F}$ & $\mathrm{I}-\mathrm{S}-\mathrm{S}$ & V-Q-F \\
\hline 32 & & & F-Y-Q & $\mathrm{I}-\mathrm{W}-\mathrm{K}$ & V-S-Q \\
\hline 33 & & & ${ }_{1} \mathrm{~F}-\mathrm{Y}-\mathrm{Y}$ & $\mathrm{I}-\mathrm{Y}-\mathrm{Q}$ & V-W-A \\
\hline 34 & & & L-G-L & K-L-E & V-W-K \\
\hline 35 & & & L-W-G & K-S-A & V-Y-Y \\
\hline 36 & & & & K-S-S & \\
\hline 37 & & & & K-S-W & \\
\hline 38 & & & & K-W-Q & \\
\hline 39 & & & & K-Y-S & \\
\hline \multirow[t]{4}{*}{40} & & & & $\mathrm{~K}-\mathrm{Y}-\mathrm{W}$ & \\
\hline & & & & $\rightarrow$ continued & \\
\hline & & & & to next & \\
\hline & & & & column & \\
\hline
\end{tabular}


Table S4. Broad-range inhibitors uncovered from the top-100 inhibitor list. The top-100 inhibitors from each metalloprotease were analyzed to compare inhibitor selectivity. Listed are the specific inhibitors that appeared in the top-100 list of all 4 metalloproteases. Identities are defined as $\mathrm{P}_{1}{ }^{\prime}-\mathrm{P}_{2}{ }^{\prime}-\mathrm{P}_{3}$.

\begin{tabular}{ll} 
No. & Inhibitor \\
\hline 1 & $\mathrm{C}_{\mathrm{p}}-\mathrm{F}-\mathrm{W}$ \\
2 & $\mathrm{~L}-\mathrm{W}-\mathrm{Y}$ \\
3 & $\mathrm{D}-\mathrm{W}-\mathrm{W}$
\end{tabular}

Table S5. $K_{D}$ analysis for Thermolysin and Anthrax LF. The data was obtained from duplicated experiment across 8 different concentrations of enzyme applied on the SMM, in a two-fold dilution series. Only data that showed significant intensities above the $50 \%$ threshold were analyzed using curve fitting. Inhibitors that did not provide proper fits (typically due to aberrant readings across the various concentration points) were also excluded. $\mathrm{K}_{\mathrm{D}}$ of inhibitors are listed in Tables S6 and S7 for Thermolysin and Anthrax LF respectively.

\begin{tabular}{cccc}
\hline $\mathrm{K}_{\mathrm{D}}$ analysis & $\begin{array}{l}\text { Filter 1. Number of } \\
\text { dataset } \\
\text { intensities above 50\% }\end{array}$ & $\begin{array}{r}\text { inhibitors of } \\
\text { exhibiting }\end{array}$ & $\begin{array}{l}\text { Filter 2. Number of inhibitors with } \\
\text { successful curve fits ( } \mathrm{r}>0.9)\end{array}$ \\
\hline Thermolysin & 109 & 43 \\
Anthrax LF & 493 & 85 \\
\hline
\end{tabular}

This large scale assessment attempts to quickly provide an overview of inhibitor potencies for a given combinatorial library. Though we were not able to obtain the $\mathrm{K}_{\mathrm{D}}$ for all the potentially strong binders (Filter 1), a significant proportion of the $\mathrm{K}_{\mathrm{D}}$ values were obtained, in a much quicker way than many other experimental approaches (eg. SPR/ Isothermal Calorimetry/Fluorescence Polarization platforms). This highlights the potential of our approach for high-throughput screening. 
Table S6. $K_{D}$ results for Thermolysin. The set of 43 inhibitors fulfilling both filter criteria of significant intensities $(>50 \%)$ and successful curve fits $(r>0.9)$ are tabulated. Inhibitors are sorted in order of increasing $\mathrm{K}_{\mathrm{D}}$. Inhibitors in bold have had their $\mathrm{K}_{\mathrm{D}} \mathrm{S}$ confirmed with SPR (Figure S10). Identities are defined as $\mathrm{P}_{1}{ }^{\prime}-\mathrm{P}_{2}{ }^{\prime}-\mathrm{P}_{3}{ }^{\prime}$.

\begin{tabular}{llcc}
\hline & & & \\
No. & Inhibitors & $\mathbf{K}_{\mathbf{D}}(\boldsymbol{\mu} \mathbf{M})$ & Curve fit (r) \\
\hline & & & \\
1 & L-Q-A & 0.89 & 0.96 \\
2 & L-H-R & 0.96 & 0.93 \\
3 & L-A-Q & 0.97 & 0.93 \\
4 & L-A-R & 1.02 & 0.96 \\
5 & L-W-S & 1.09 & 0.96 \\
6 & L-W-K & 1.13 & 0.94 \\
7 & L-M-V & 1.28 & 0.97 \\
8 & L-F-Y & 1.35 & 0.92 \\
9 & L-M-A & 1.45 & 0.95 \\
10 & L-P-W & 1.50 & 0.92 \\
11 & L-R-P & 1.72 & 0.98 \\
12 & L-Q-Q & 1.76 & 0.96 \\
13 & L-R-R & 1.90 & 0.92 \\
14 & L-A-P & 1.99 & 0.90 \\
15 & L-M-R & 2.04 & 0.98 \\
16 & L-I-W & 2.05 & 0.97 \\
17 & L-A-T & 2.05 & 0.92 \\
18 & L-A-W & 2.14 & 0.95 \\
19 & L-L-C & 2.14 & 0.95 \\
20 & L-H-Y & 2.17 & 0.96 \\
21 & L-R-M & 2.25 & 0.97 \\
22 & L-Q-K & 2.36 & 0.91 \\
23 & L-I-F & 2.37 & 0.94 \\
24 & L-Y-R & 2.39 & 0.94 \\
25 & L-W-E & 2.52 & 0.94 \\
26 & L-W-Q & 2.54 & 0.97 \\
27 & L-W-F & 2.70 & 0.94 \\
28 & L-W-L & 2.74 & 0.97 \\
29 & L-R-V & 2.77 & 0.92 \\
30 & L-M-Y & 2.86 & 0.96 \\
31 & L-H-W & 2.94 & 0.95 \\
32 & L-R-W & 3.07 & 0.93 \\
33 & L-K-A & 3.07 & 0.98 \\
34 & L-S-N & 3.09 & 0.93 \\
35 & L-M-K & 3.23 & 0.94 \\
36 & L-L-Y & 3.58 & 0.93 \\
37 & L-H-P & 3.61 & 0.90 \\
38 & L-R-G & 3.93 & 0.98 \\
39 & L-T-Q & 3.98 & 0.98 \\
40 & L-Y-G & 4.01 & 0.97 \\
41 & L-T-R & 4.24 & 0.95 \\
42 & L-I-V & 4.37 & 0.92 \\
43 & L-M-W & 4.69 & 0.97 \\
& & & \\
\hline & & & \\
\hline
\end{tabular}


Table S7. $K_{D}$ results for Anthrax LF. The set of 85 inhibitors fulfilling both filter criteria of significant intensities $(>50 \%)$ and successful curve fits $(r>0.9)$ are tabulated. Identities are defined as $\mathrm{P}_{1}{ }^{\prime}-\mathrm{P}_{2}{ }^{\prime}$ $\mathrm{P}_{3}{ }_{3}$. Inhibitors are sorted in order of increasing $\mathrm{K}_{\mathrm{D}}$. Inhibitors in bold have also been tested for $\mathrm{IC}_{50}$ (Table 3).

\begin{tabular}{|c|c|c|c|c|c|c|c|}
\hline No. & Inhibitors & $\mathbf{K}_{\mathbf{D}}(\boldsymbol{\mu} \mathbf{M})$ & Curve fit (r) & No. & Inhibitors & $\mathbf{K}_{\mathbf{D}}(\boldsymbol{\mu} \mathbf{M})$ & Curve fit (r) \\
\hline 1 & ${ }_{1} \mathrm{~F}-\mathrm{L}-\mathrm{E}$ & 0.71 & 0.99 & 43 & L-W-R & 2.96 & 0.97 \\
\hline 2 & F-W-L & 0.71 & 0.92 & 44 & L-L-T & 2.97 & 0.96 \\
\hline 3 & I-Y-L & 0.74 & 0.92 & 45 & I-A-H & 3.03 & 0.96 \\
\hline 4 & ${ }_{1}$ F-F-L & 0.81 & 0.93 & 46 & K-Q-A & 3.04 & 0.96 \\
\hline 5 & V-L-E & 0.85 & 0.91 & 47 & L-N-H & 3.18 & 0.97 \\
\hline 6 & F-W-S & 0.86 & 0.96 & 48 & F-S-H & 3.20 & 0.97 \\
\hline 7 & $\mathrm{~S}_{\mathrm{f}}-\mathrm{L}-\mathrm{W}$ & 1.06 & 0.94 & 49 & ${ }_{1} F-E-Q$ & 3.22 & 0.94 \\
\hline 8 & D-Q -L & 1.14 & 0.93 & 50 & D-A-S & 3.26 & 0.95 \\
\hline 9 & ${ }_{1} \mathrm{~F}-\mathrm{S}-\mathrm{Q}$ & 1.21 & 0.96 & 51 & K-Q-Y & 3.28 & 0.95 \\
\hline 10 & I-S-A & 1.22 & 0.93 & 52 & $\mathrm{~S}_{\mathrm{f}}-\mathrm{L}-\mathrm{F}$ & 3.29 & 0.98 \\
\hline 11 & ${ }_{1} \mathrm{~F}-\mathrm{E}-\mathrm{S}$ & 1.23 & 0.90 & 53 & L-G-Y & 3.31 & 0.98 \\
\hline 12 & D-E-S & 1.26 & 0.90 & 54 & $\mathrm{~S}_{\mathrm{f}}-\mathrm{S}-\mathrm{Q}$ & 3.35 & 0.92 \\
\hline 13 & D-S-Y & 1.28 & 0.92 & 55 & ${ }_{1}$ F-E-K & 3.44 & 0.98 \\
\hline 14 & F-L-F & 1.30 & 0.95 & 56 & $\mathrm{~S}_{\mathrm{f}}-\mathrm{Q}-\mathrm{W}$ & 3.47 & 0.94 \\
\hline 15 & lF-L-W & 1.34 & 0.94 & 57 & K-S-S & 3.55 & 0.95 \\
\hline 16 & L-Y-Y & 1.36 & 0.99 & 58 & D-L-L & 3.56 & 0.95 \\
\hline 17 & ${ }_{1} \mathrm{~F}-\mathrm{S}-\mathrm{E}$ & 1.45 & 0.93 & 59 & L-P-M & 3.65 & 0.96 \\
\hline 18 & F-S-A & 1.57 & 0.93 & 60 & I-S-Q & 3.68 & 0.97 \\
\hline 19 & K-S-L & 1.63 & 0.95 & 61 & $\mathrm{~L}-\mathrm{V}-\mathrm{T}$ & 3.73 & 0.95 \\
\hline 20 & D-E-E & 1.69 & 0.92 & 62 & L-G-F & 3.84 & 0.97 \\
\hline 21 & D-S-L & 1.80 & 0.98 & 63 & L-D-L & 3.86 & 0.95 \\
\hline 22 & $\mathrm{~S}_{\mathrm{f}}-\mathrm{A}-\mathrm{K}$ & 1.86 & 0.94 & 64 & L-S-Y & 3.96 & 0.97 \\
\hline 23 & ${ }_{1} \mathrm{~F}-\mathrm{A}-\mathrm{H}$ & 1.91 & 0.97 & 65 & K-A-E & 4.03 & 0.97 \\
\hline 24 & ${ }_{1}$ F-L-L & 1.99 & 0.96 & 66 & L-M-F & 4.20 & 0.98 \\
\hline 25 & L-D-C & 2.02 & 0.97 & 67 & ${ }_{1} \mathrm{~F}-\mathrm{E}-\mathrm{A}$ & 4.27 & 0.96 \\
\hline 26 & ${ }_{1} \mathrm{~F}-\mathrm{Q}-\mathrm{Y}$ & 2.03 & 0.98 & 68 & L-P-E & 4.50 & 0.93 \\
\hline 27 & D-A-Q & 2.08 & 0.92 & 69 & L-E-P & 4.53 & 0.98 \\
\hline 28 & F-E-A & 2.13 & 0.94 & 70 & L-I-R & 4.66 & 0.98 \\
\hline 29 & K-E-Q & 2.14 & 0.95 & 71 & D-E-W & 4.73 & 0.97 \\
\hline 30 & L-F-P & 2.16 & 0.98 & 72 & $D-A-Y$ & 4.76 & 0.97 \\
\hline 31 & $S_{\mathrm{f}}-\mathrm{Q}-\mathrm{Y}$ & 2.17 & 0.93 & 73 & L-Q-F & 4.87 & 0.96 \\
\hline 32 & K-Q-E & 2.21 & 0.99 & 74 & $\mathrm{~L}-\mathrm{W}-\mathrm{N}$ & 5.08 & 0.99 \\
\hline 33 & I-Q-H & 2.30 & 0.94 & 75 & F-A-Q & 5.32 & 0.96 \\
\hline 34 & F-Q-A & 2.34 & 0.96 & 76 & L-W-F & 5.41 & 0.97 \\
\hline 35 & ${ }_{1}$ F-E-L & 2.42 & 0.98 & 77 & I-Q-S & 5.55 & 0.98 \\
\hline 36 & ${ }_{1} \mathrm{~F}-\mathrm{E}-\mathrm{H}$ & 2.50 & 0.97 & 78 & L-E-H & 5.61 & 0.97 \\
\hline 37 & K-L-S & 2.53 & 0.96 & 79 & D-K-Q & 5.62 & 0.92 \\
\hline 38 & I-A-A & 2.60 & 0.92 & 80 & V-Q-H & 6.23 & 0.96 \\
\hline 39 & K-E-E & 2.72 & 0.98 & 81 & L-Q-M & 6.30 & 0.98 \\
\hline 40 & ${ }_{1} \mathrm{~F}-\mathrm{Q}-\mathrm{F}$ & 2.76 & 0.97 & 82 & V-A-Y & 6.75 & 0.98 \\
\hline 41 & ${ }_{1} \mathrm{~F}-\mathrm{S}-\mathrm{H}$ & 2.85 & 0.93 & 83 & L-V-C & 7.16 & 0.99 \\
\hline 42 & $\mathrm{~S}_{\mathrm{f}}-\mathrm{A}-\mathrm{Y}$ & 2.93 & 0.97 & 84 & V-S-K & 7.84 & 0.97 \\
\hline \multicolumn{4}{|c|}{$\rightarrow$ continued to next column } & 85 & L-P-A & 8.41 & 0.99 \\
\hline
\end{tabular}


Table S8. Selectivity of $\mathbf{I}$-F-L-L against panel of enzymes.

\begin{tabular}{cccc}
\hline \multirow{2}{*}{ F-F-L } & Primary Screen & \multicolumn{2}{c}{ Secondary Screening } \\
\cline { 2 - 4 } & $\begin{array}{c}\text { Microarray Inhibition } \\
\text { Potency/ \% }\end{array}$ & $\mathrm{IC}_{50} / \mu \mathrm{M}$ & $\mathrm{K}_{\mathrm{D}}$ on SMM/ $\mu \mathrm{M}$ \\
\hline Anthrax LF & 90.0 & 2.0 & 0.81 \\
Thermolysin & 18.2 & $>25$ & n.d. \\
Collagenase & 64.9 & $>25$ & n.d. \\
Carboxypeptidase & 47.8 & n.d. & n.d. \\
\hline
\end{tabular}

n.d. - Not Determined 


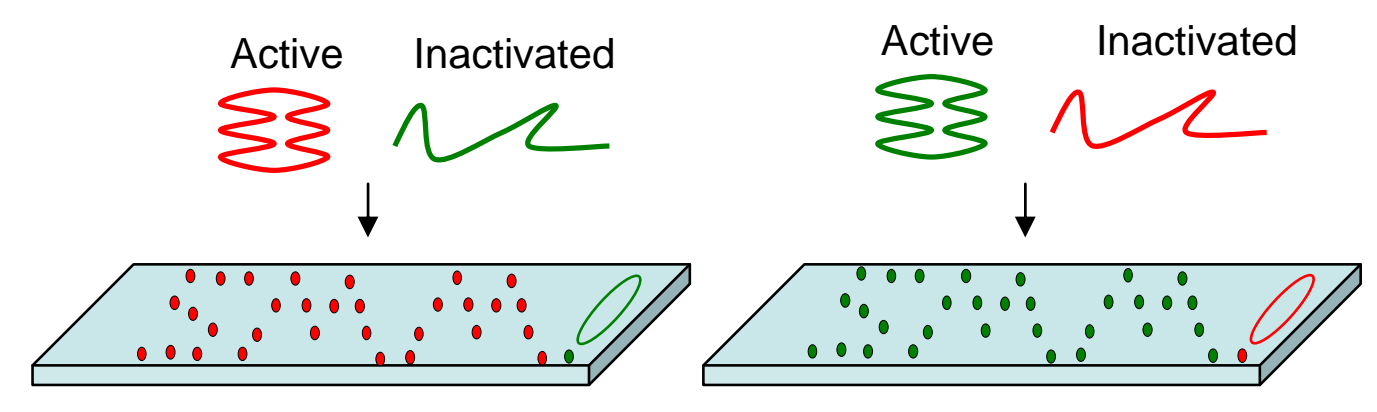

Figure S1. Reciprocal labeling and application strategy for activity-dependent high-throughput microarray screening. Cy3 labeled protein is false colored in green, while Cy5 labeled protein is false colored in red. Normalized signals from the active protein channel constitute real binding profiles on the SMM. Non-specific binding from denatured or inactive proteins can be readily identified and corrected (i.e. exclamation point).

\section{Intensities using Spotted Dye}

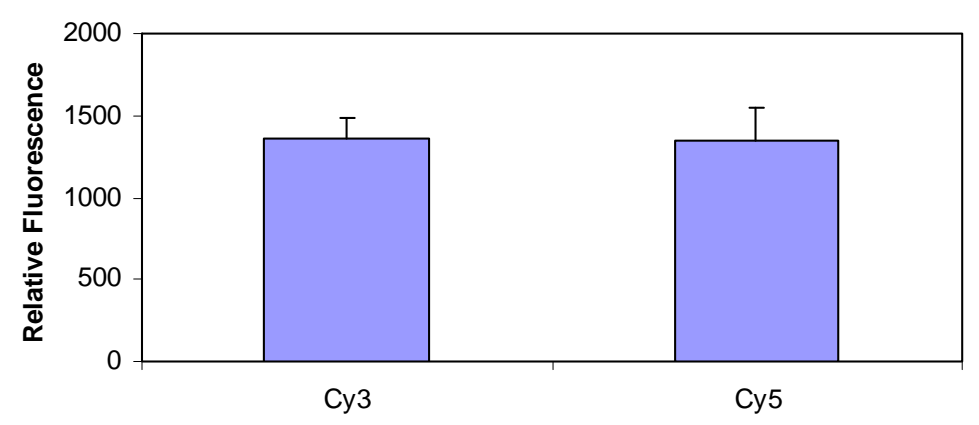

Figure S2. Equivalent concentrations of $\mathrm{Cy} 3$ and $\mathrm{Cy} 5$ dye were spotted and scanned using the ArrayWoRx microarray scanner. Highly correlated values were obtained across the different dye channels. The error bar denotes standard deviation over 5 replicates. 
<smiles>CNC(=O)[C@H](Cc1cc2ccccc2[nH]1)NC(=O)C(CC(=O)NO)CC(C)C</smiles><smiles>CNC(=O)[C@H](NC(=O)[C@H](CC(C)C)[C@H](O)C(=O)NO)C(C)(C)C</smiles><smiles>CNC(=O)C(Cc1ccccc1)NC(=O)C(CC(C)C)C(Sc1cccs1)C(=O)NO</smiles>

Figure S3. Structures of GM6001, Marimastat and Batismastat.

Microplate Profile

$\mathbf{P}^{\prime}$

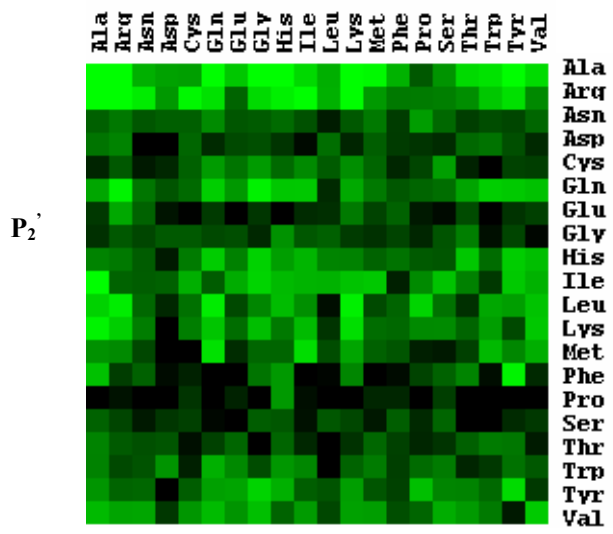

SMM Profile

$\mathbf{P}_{3}^{\prime}$

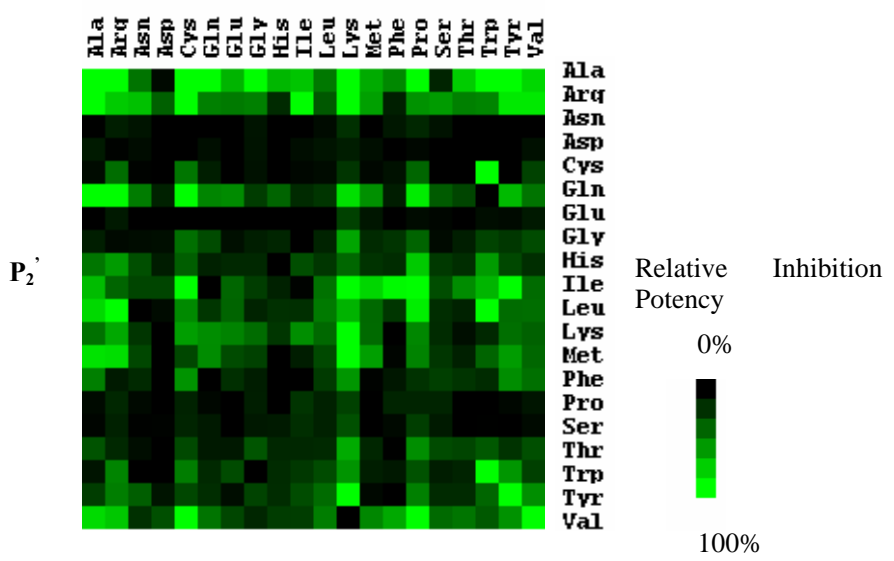

Figure S4. The 400-member $\mathrm{P}_{1}$, L sub-library was screened using microplate and compared with the fingerprint obtained using SMM. The most potent inhibitors are displayed in green, according to the scale shown inset.

Performing the assay in solution phase involves reaction dynamics that may not be perfectly replicable on solid support, due to the static visualization of intensity signals on microarray, as well as the modes of ligand binding, which is complicated with bulk effects, slower kinetics and ambient analyte effects. ${ }^{4}$ Nevertheless, mostly consistent fingerprints were obtained from the two experiments, with good similarity in their profiles (Pearsson Correlation, $r=0.54$ ). Of note, under varying treatments and washing conditions of the SMM the correlations fluctuate (data not shown). This highlights the importance of careful optimization and handling procedures to extract the most accurate and meaningful activity-dependent profiles using SMM. 


\section{Thermolysin}

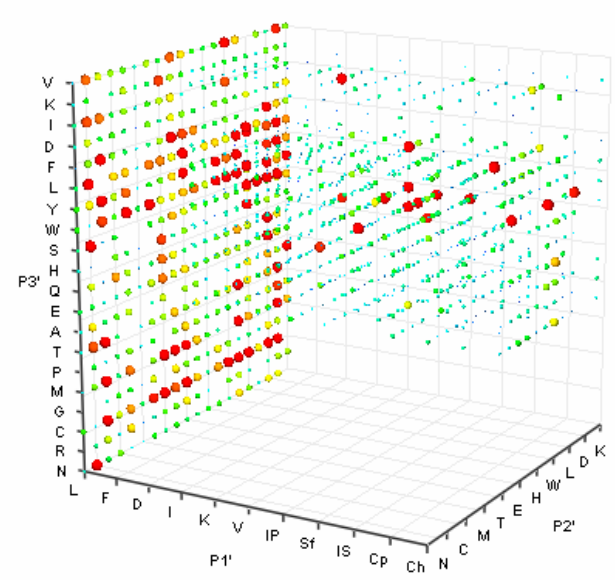

III. Carboxypeptidase

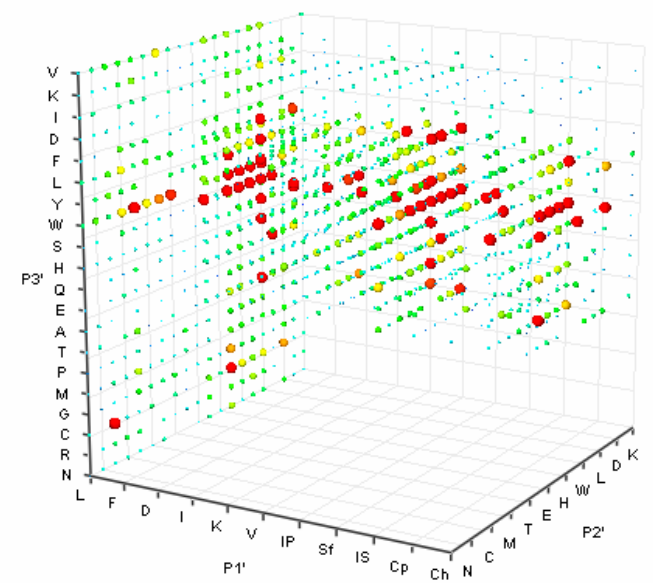

II. Collagenase

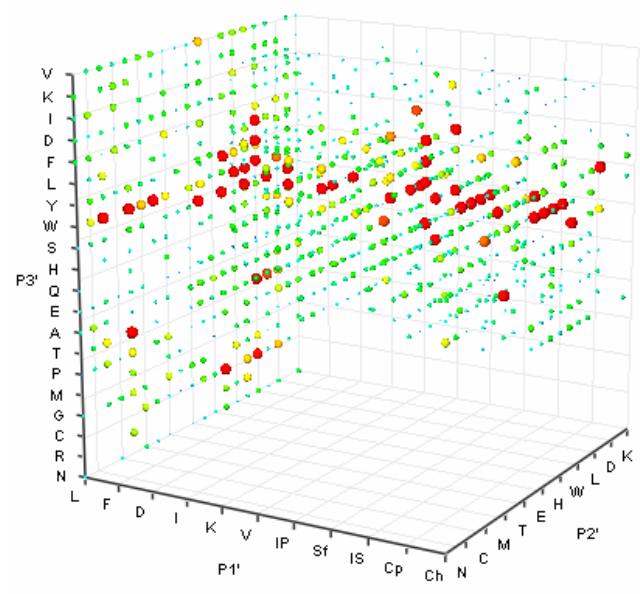

IV. Anthrax LF

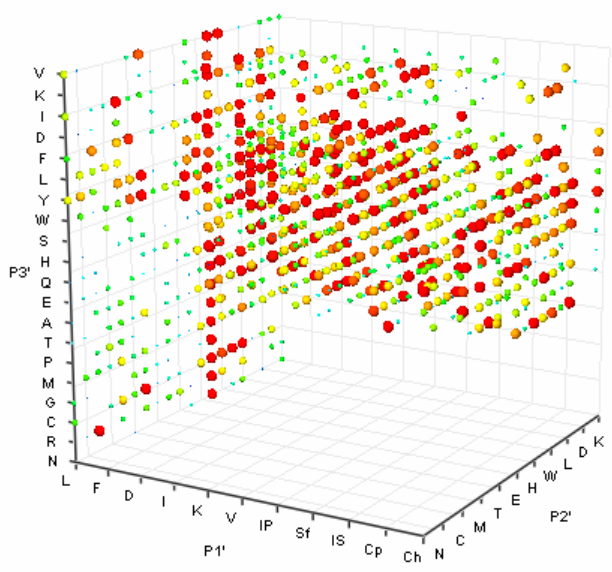

Figure S5. The data combined from both reciprocal experiments were presented in a 3D cube plot for four enzymes in the panel (the cube plot for thermolysin is also displayed in Figure 1C of the maintext). Each sphere coordinate in the plot corresponds to the $\mathrm{P}_{1}{ }^{\prime}, \mathrm{P}_{2}{ }^{\prime}$ and $\mathrm{P}_{3}$ ' identities of the inhibitors allowing binding intensities of all 1,400 cognate members to be visualized simultaneously. Relative potency is indicated by both the color spectrum (blue - least potent, red - most potent) as well as by size of the sphere (small - least potent, big - most potent). Only data that was highly discordant between the reciprocal experiments were excluded. Nevertheless a significant proportion of the data, $70 \%$ of the data in the case of collagenase, $75 \%$ for Anthrax LF, $90 \%$ for carboxypeptidase and $95 \%$ for thermolysin were consistently reproduced across the reciprocal channels. Statistical correlation between the duplicated experiments was strong $(r>0.8)$ with thermolysin providing the highest correlation measure of $r=0.92$. Original data is provided in the Supplementary Excel file. 
$\mathrm{P}_{1}{ }^{\prime}$

Anthrax LF
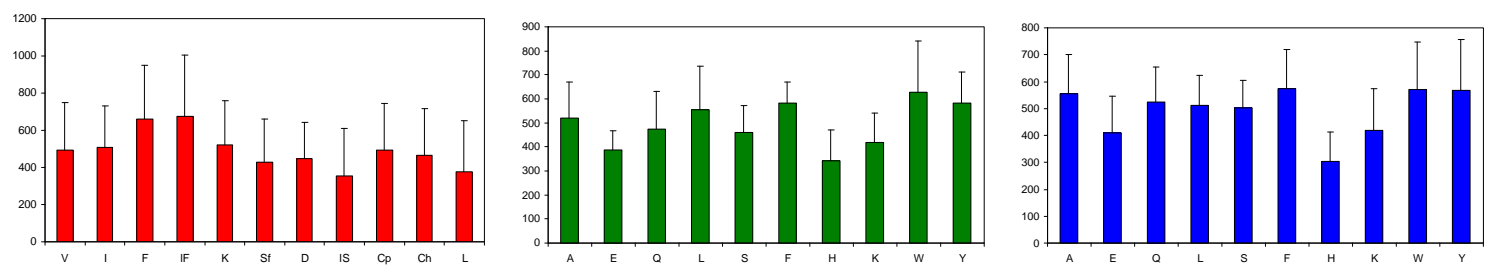

Thermolysin
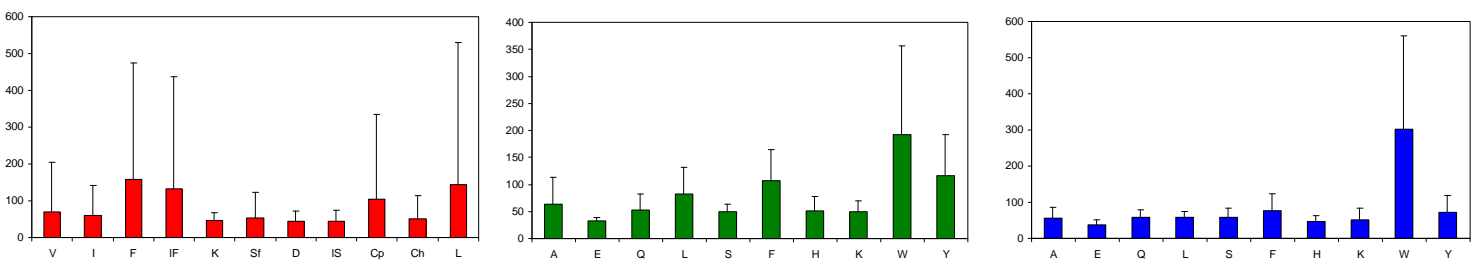

Collagenase
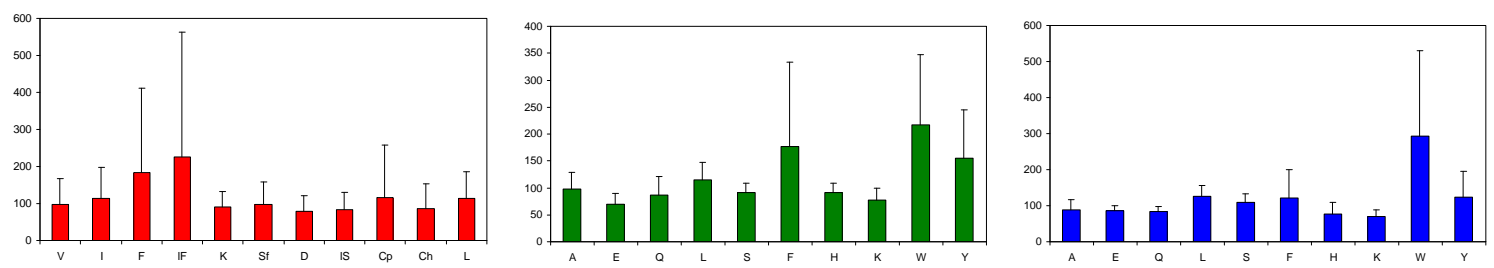

Carboxypeptidase
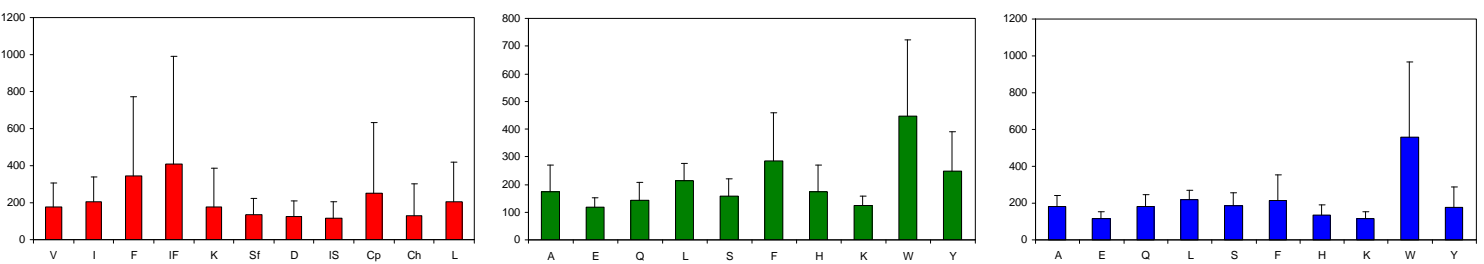

Figure S6. Averaged inhibition contributions across permuted $\mathrm{P}_{1}{ }^{\prime}, \mathrm{P}_{2}{ }^{\prime}$ and $\mathrm{P}_{3}$ ' positions. Each bar represents averaged inhibition across inhibitors in the library presenting the relevant residue. The error bar denotes the standard deviation across each group of inhibitors. Though information was lost by averaging because of consolidation of individual inhibitor sequences into general categories, this analysis provided for a broader analysis of the data and a snapshot view of the results, depicting each residues overall bearing to potency. 
A

Combined (232)

Thermolysin (100)

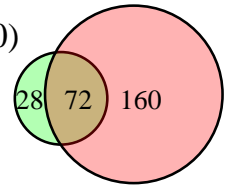

Combined (225)
Combined (229)

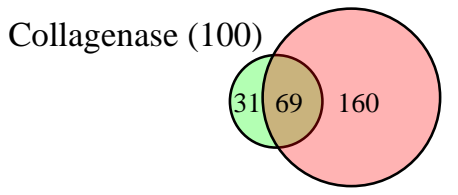

Combined (185)
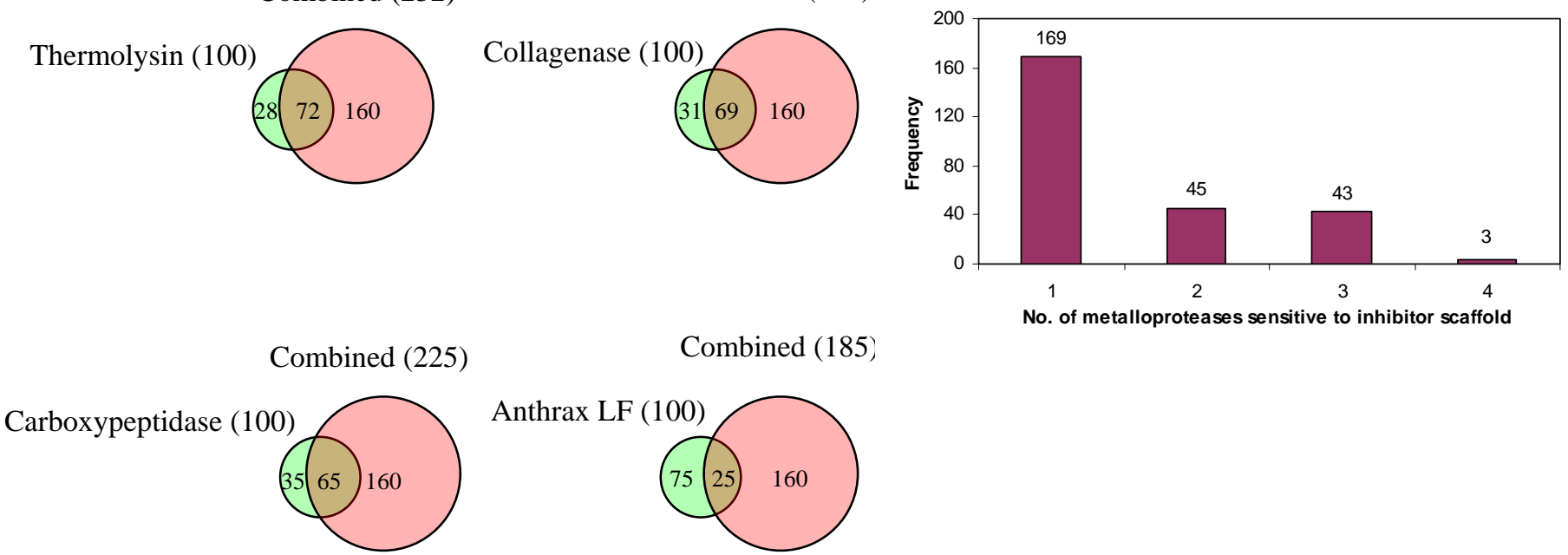

Figure S7. Distribution of the top-100 inhibitors. See Table S3 for more details. (A) Venn diagrams representing distributions of top-100 inhibitors for each metalloprotease. Regions in green highlight uniquely binding potent inhibitors, while regions in orange indicate broadly selective potent inhibitors. The values represent total inhibitor numbers in each category. Specifically, Anthrax LF presented the largest number of selective inhibitors - a total of 75 - from the 100 selected. (B) A total of 169 inhibitors (65\%) out of the 260 unique scaffolds in the top-100 set were selective against individual metalloproteases (Table S4). 3 broadly selective inhibitors were uncovered that bound potently with all 4 metalloproteases (Table S5). We also found that a series of 45 and 43 inhibitor sequences from the top 100 lists that targeted combinations of 2 and 3 metalloproteases, respectively. 


\begin{tabular}{|c|c|c|}
\hline & $\begin{array}{cc}14 & 15 \\
234567890123456789012345 A \\
\# \# \quad \#\end{array}$ & $\begin{array}{c}16 \quad 17 \\
678901234567890123456 \\
\#\end{array}$ \\
\hline \multicolumn{3}{|c|}{ 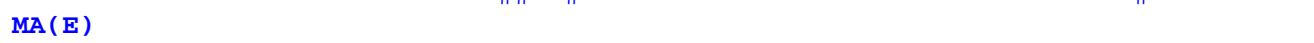 } \\
\hline Thermolysin & PLSGGIDVVAHELTHAVTDYTAGL - & IYQNESGAINEAISDIFGTLV \\
\hline Collagenase & STYTLEELFRHEYTHYLQGRYAVPG & YDNDRLTWYEEGGAELFA \\
\hline \multirow[t]{2}{*}{ Anthrax LF } & ELRNDSEGFIHEFGHAVDDYAGYLL & LTSYGRTNEAEFFAEAFRLMH \\
\hline & $\begin{array}{c}23 \\
123456789012345678901 \\
\#\end{array}$ & \\
\hline \multicolumn{3}{|l|}{$M A(E)$} \\
\hline \multirow{4}{*}{$\begin{array}{l}\text { Thermolysin } \\
\text { Collagenase } \\
\text { Anthrax LF }\end{array}$} & YTGTQDNGGVHINSGIINKAA & \\
\hline & & \\
\hline & $\begin{array}{lll}18 & 19 & 20\end{array}$ & 23 \\
\hline & $\begin{array}{c}901234567890123456789012 \\
\# \quad \#\end{array}$ & $\begin{array}{c}789012345678901234567 \\
\#\end{array}$ \\
\hline \multicolumn{3}{|l|}{ MC } \\
\hline \multirow[t]{3}{*}{ Carboxypeptidase } & RPAIWIDTGIHSREWVTQASGVWF & AFTHSTNRMWRKTRSHTAGSL \\
\hline & 30 & 37 \\
\hline & $\begin{array}{c}678901234567890123456 \\
\#\end{array}$ & $\begin{array}{c}012345678901234567890 \\
\#\end{array}$ \\
\hline \multicolumn{3}{|c|}{ - } \\
\hline Carboxypeptidase & HGNIKAFISIHSYSQLLMYPY & SQGIKYSFTFELRDTGRYGFL \\
\hline
\end{tabular}

Figure S8. Protein sequence alignment of the enzymes used in this study.

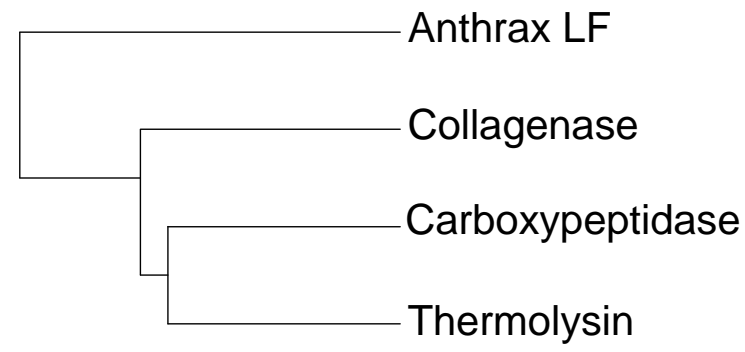

Figure S9. Cladograms of metalloprotease based on SMM inhibitor fingerprints. The panel was clustered according to the complete dataset with the 1,400 inhibitors. Average linkage clustering was performed.

This relationship was conveniently discernable using the SMM platform, and the utility of such a functional classification may not be easily replicated using existing schemes of substrate preference or primary sequence information alone, thereby highlighting the value of the presented approach for activity-dependent classification. 


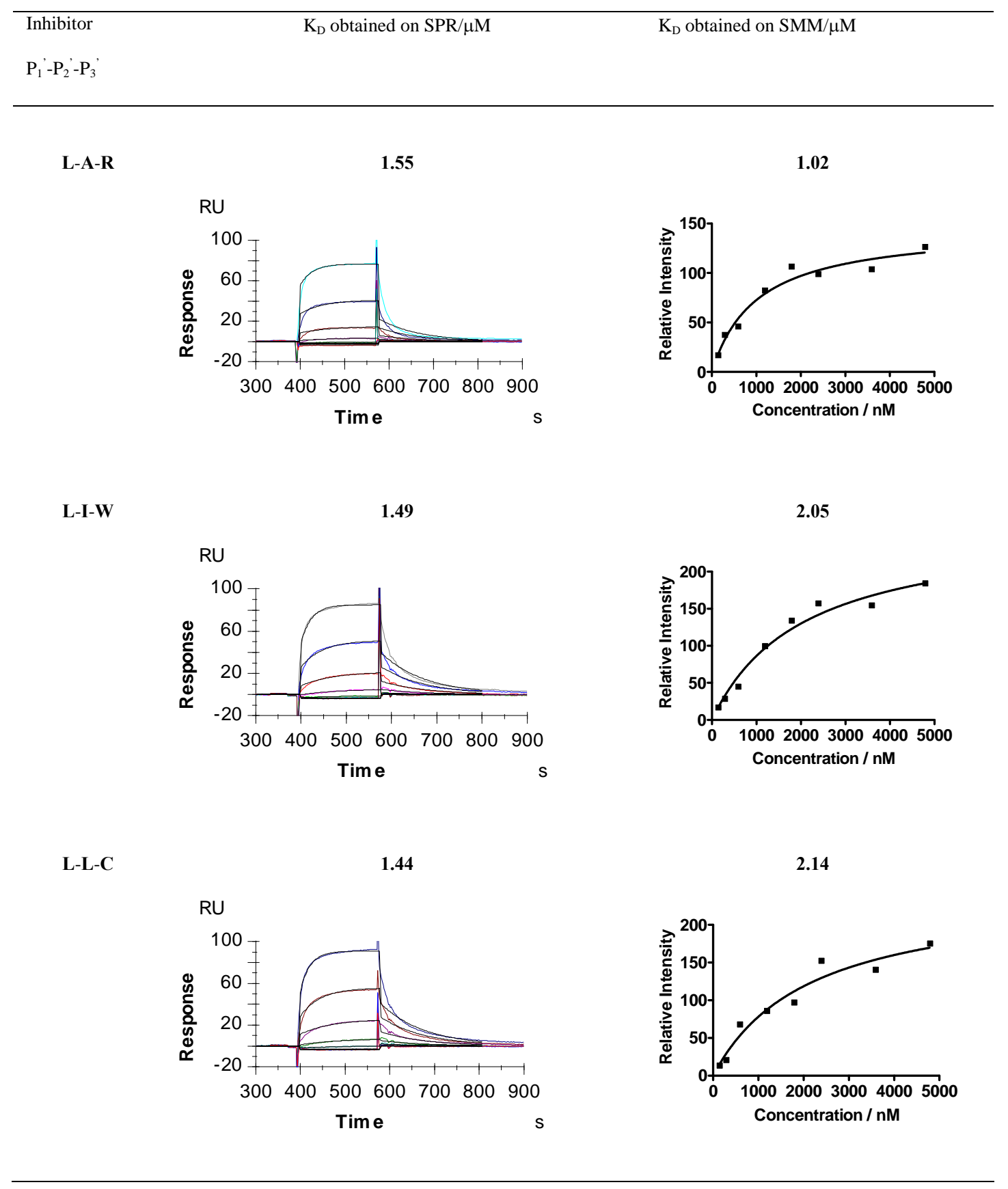

Figure S10. SPR was used to confirm selected $K_{D}$ values obtained against thermolysin on the SMM. A very good consistency was seen with the values obtained on both platforms (less than 1-fold difference in value). 


\section{IC $_{50}$ Curves}

ALF

IF-F-L IC50 $=2.0 \mathrm{uM}$

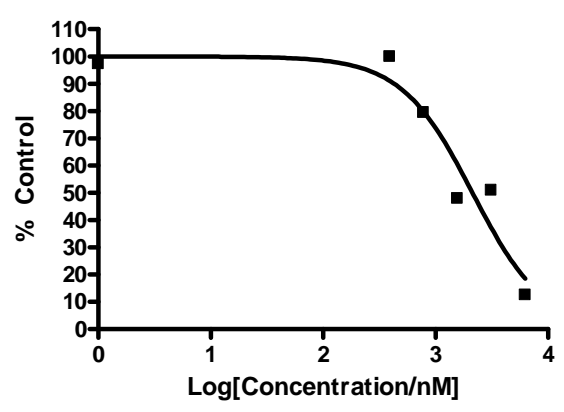

ALF

$F-W-L$ IC50 $=2.2$ uM

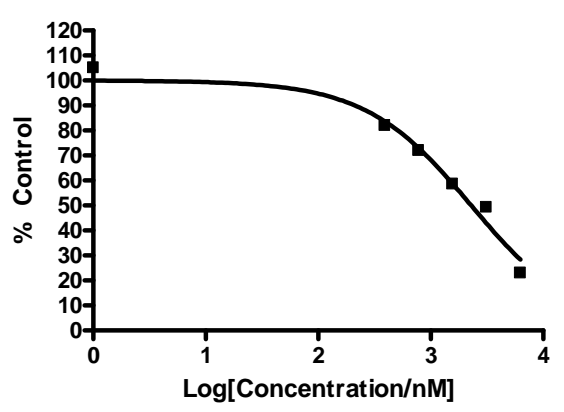

ALF

I-Y-L IC50 $=5.5 \mathrm{uM}$

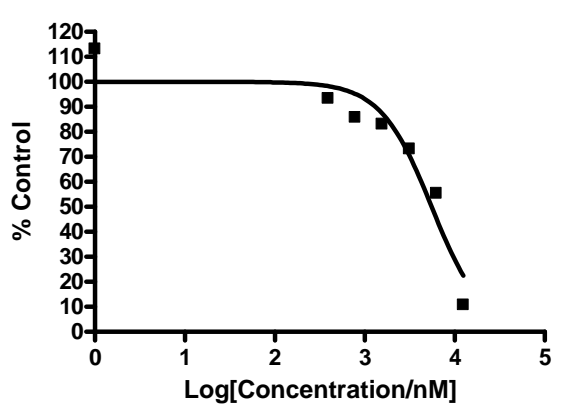

ALF

F-W-S IC50 $=7.9$ uM

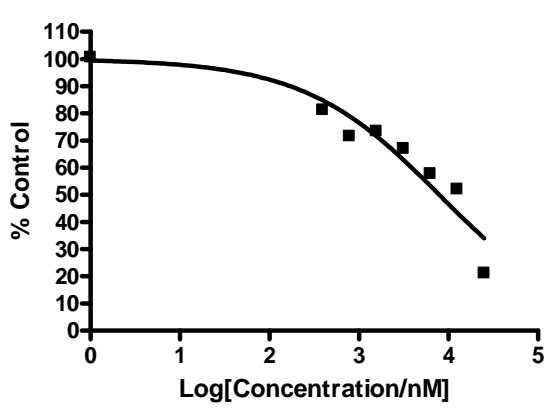

$K_{D}$ Curves

ALF

LF-F-L KD $=0.81$ uM

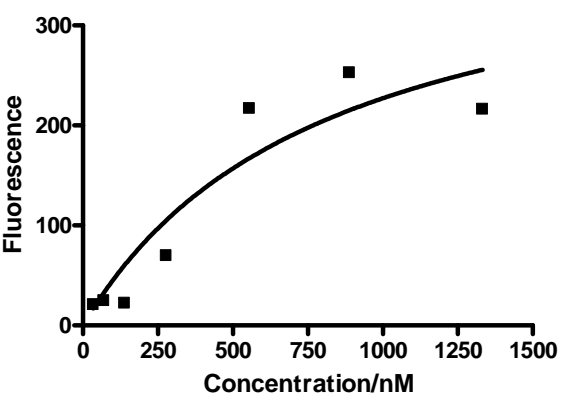

ALF

$F-W-L ~ K D=0.71 ~ u M$

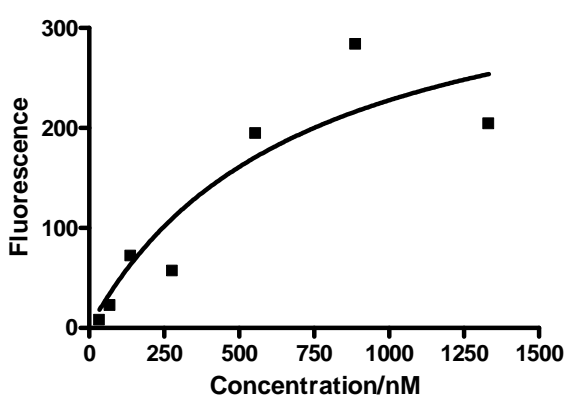

ALF

I-Y-L KD $=0.74$ uM

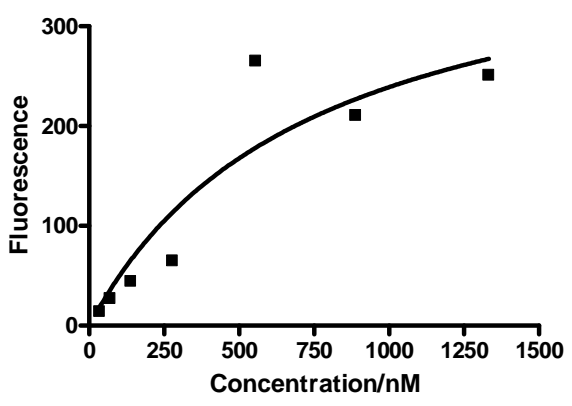

ALF

F-W-S KD $=0.86 \mathrm{uM}$

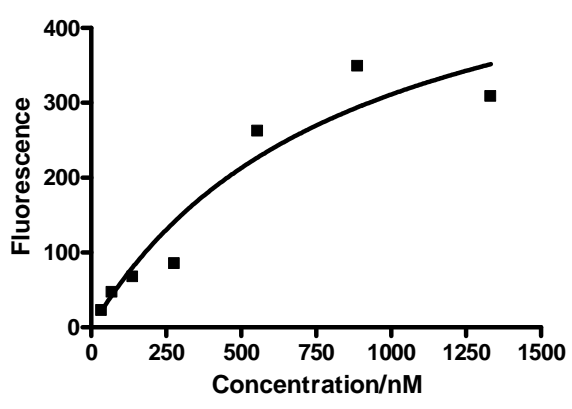


ALF

I-A-A IC50 $=14.0 \mathrm{uM}$

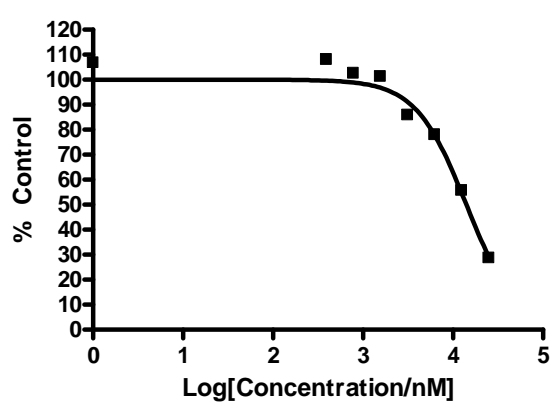

ALF

L-P-A IC50 = 16.3 uM

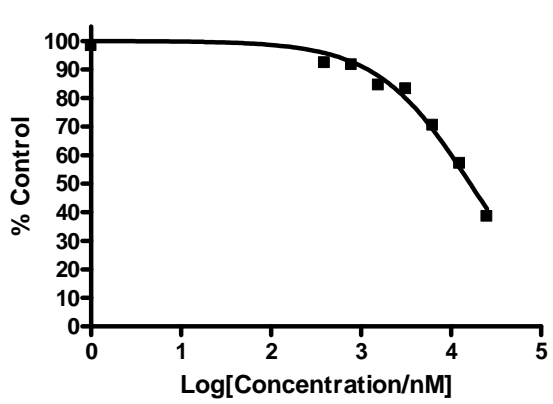

ALF

L-D-C IC50 $=29.6$ uM

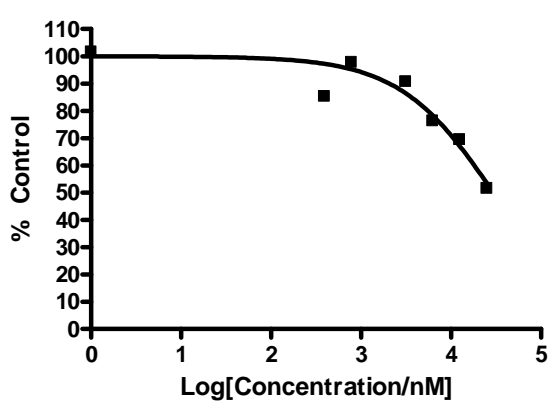

ALF

I-A-A KD = 2.6 uM

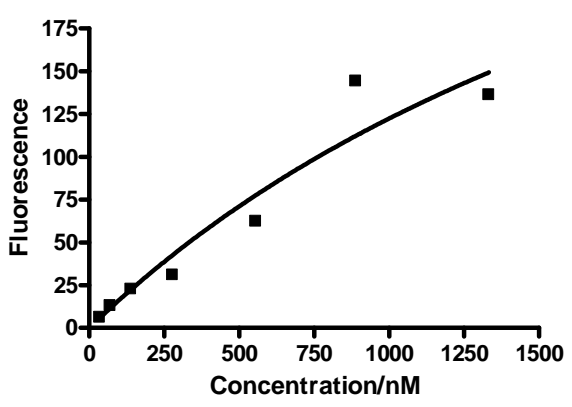

ALF

L-P-A KD $=8.43$ uM

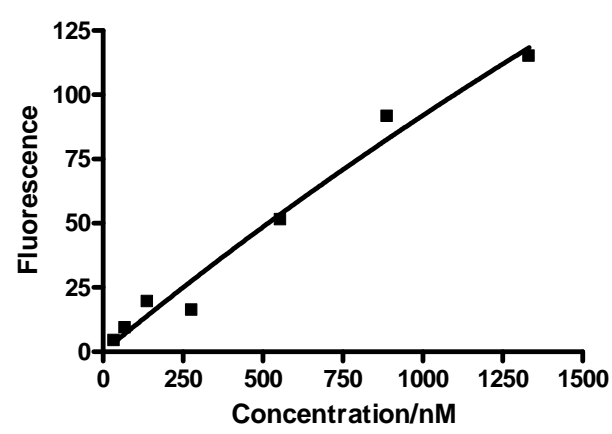

ALF

L-D-C KD $=2.0 \mathrm{uM}$

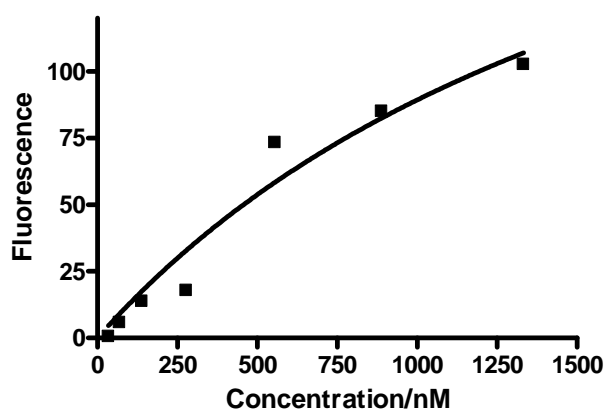

Figure S11. $\mathrm{IC}_{50}$ and $\mathrm{K}_{\mathrm{D}}$ curves for selected inhibitors with Anthrax LF. A panel of inhibitors for Anthrax LF was selected and evaluated for quantitative binding parameters.

\section{References}

1. Fujii, K.; Zhu, G.; Liu, Y.; Hallam, J.; Chen, L.; Herrero, J.; Shaw, S. Proc. Natl. Acad. Sci. USA, 2004, 101, 13744-13749.

2. $\quad$ www.//merops.sanger.ac.uk; Rawlings, N. D.; Morton, F. R.; Barrett, A. J.; Nucleic Acids Res. 2006, 34, D270-D272.

3. Chenna, R.; Sugawara, H.; Koike, T.; Lopez, R.; Gibson, T. J.; Higgins, D. G.; Thompson, J. D. Nucleic Acids Res. 2003, 31, 3497-3500.

4. Templin. M. F.; Stoll. D.; Schrenk, M. ; Traub, P. C.; Vohringer, C. F.; Joos. T. O. Trends Biotechnol. 2002, 20, 160-166. 